\title{
Article \\ Automatic COVID-19 Detection Using Exemplar Hybrid Deep Features with X-ray Images
}

\author{
Prabal Datta Barua ${ }^{1}$, Nadia Fareeda Muhammad Gowdh ${ }^{2}{ }^{-0}$, Kartini Rahmat ${ }^{2}$, Norlisah Ramli ${ }^{2}$, Wei Lin Ng ${ }^{2}$, \\ Wai Yee Chan ${ }^{2}\left(\mathbb{D}\right.$, Mutlu Kuluozturk ${ }^{3}$, Sengul Dogan $4, * \mathbb{(}$, Mehmet Baygin ${ }^{5}$, Orhan Yaman ${ }^{4}{ }^{\circ}$, Turker Tuncer ${ }^{4}{ }^{\oplus}$, \\ Tao Wen ${ }^{6}$, Kang Hao Cheong ${ }^{6, *(1)}$ and U. Rajendra Acharya ${ }^{7,8,9}$ (])
}

check for

updates

Citation: Barua, P.D.;

Muhammad Gowdh, N.F.; Rahmat, K.

Ramli, N.; Ng, W.L.; Chan, W.Y.;

Kuluozturk, M.; Dogan, S.; Baygin, M.;

Yaman, O.; et al. Automatic COVID-19

Detection Using Exemplar Hybrid

Deep Features with X-ray Images. Int.

J. Environ. Res. Public Health 2021, 18,

8052. https://doi.org/10.3390/

ijerph18158052

Academic Editor: Paul B. Tchounwou

Received: 24 June 2021

Accepted: 22 July 2021

Published: 29 July 2021

Publisher's Note: MDPI stays neutral with regard to jurisdictional claims in published maps and institutional affiliations.

Copyright: (c) 2021 by the authors. Licensee MDPI, Basel, Switzerland. This article is an open access article distributed under the terms and conditions of the Creative Commons Attribution (CC BY) license (https:/ / creativecommons.org/licenses/by/ $4.0 /)$
1 School of Management \& Enterprise, University of Southern Queensland, Toowoomba 2550, Australia; Prabal.Barua@usq.edu.au

2 Department of Biomedical Imaging, Faculty of Medicine, University of Malaya, Kuala Lumpur 50603, Malaysia; nadia_fareeda@ummc.edu.my (N.F.M.G.); kartini@ummc.edu.my (K.R.); norlisah@ummc.edu.my (N.R.); wei.lin@ummc.edu.my (W.L.N.); waiyeec@ummc.edu.my (W.Y.C.)

3 Department of Pulmonology Clinic, Firat University Hospital, Firat University, Elazig 23119, Turkey; mutlukuluozturk@hotmail.com

4 Department of Digital Forensics Engineering, College of Technology, Firat University, Elazig 23119, Turkey; orhanyaman@firat.edu.tr (O.Y.); turkertuncer@firat.edu.tr (T.T.)

5 Department of Computer Engineering, College of Engineering, Ardahan University, Ardahan 75000, Turkey mehmetbaygin@ardahan.edu.tr

6 Science, Mathematics and Technology Cluster, Singapore University of Technology and Design, 8 Somapah Road, Singapore S485998, Singapore; tao_wen@mymail.sutd.edu.sg

7 Department of Electronics and Computer Engineering, Ngee Ann Polytechnic, Singapore S599489, Singapore; aru@np.edu.sg

8 Department of Biomedical Engineering, School of Science and Technology, Singapore University of Social Sciences, Singapore S599494, Singapore

9 Department of Biomedical Informatics and Medical Engineering, Asia University, Taichung 41354, Taiwan

* Correspondence: sdogan@firat.edu.tr (S.D.); kanghao_cheong@sutd.edu.sg (K.H.C.)

Abstract: COVID-19 and pneumonia detection using medical images is a topic of immense interest in medical and healthcare research. Various advanced medical imaging and machine learning techniques have been presented to detect these respiratory disorders accurately. In this work, we have proposed a novel COVID-19 detection system using an exemplar and hybrid fused deep feature generator with X-ray images. The proposed Exemplar COVID-19FclNet9 comprises three basic steps: exemplar deep feature generation, iterative feature selection and classification. The novelty of this work is the feature extraction using three pre-trained convolutional neural networks (CNNs) in the presented feature extraction phase. The common aspects of these pre-trained CNNs are that they have three fully connected layers, and these networks are AlexNet, VGG16 and VGG19. The fully connected layer of these networks is used to generate deep features using an exemplar structure, and a nine-feature generation method is obtained. The loss values of these feature extractors are computed, and the best three extractors are selected. The features of the top three fully connected features are merged. An iterative selector is used to select the most informative features. The chosen features are classified using a support vector machine (SVM) classifier. The proposed COVID19 FclNet 9 applied nine deep feature extraction methods by using three deep networks together. The most appropriate deep feature generation model selection and iterative feature selection have been employed to utilise their advantages together. By using these techniques, the image classification ability of the used three deep networks has been improved. The presented model is developed using four X-ray image corpora (DB1, DB2, DB3 and DB4) with two, three and four classes. The proposed Exemplar COVID-19FclNet9 achieved a classification accuracy of $97.60 \%, 89.96 \%, 98.84 \%$ and $99.64 \%$ using the SVM classifier with 10-fold cross-validation for four datasets, respectively. Our developed Exemplar COVID-19FclNet9 model has achieved high classification accuracy for all four databases and may be deployed for clinical application. 
Keywords: Exemplar COVID-19FclNet9; deep feature generation; transfer learning; COVID-19 detection; iterative NCA

\section{Introduction}

The COVID-19 pandemic is an ongoing global pandemic caused by the severe acute respiratory syndrome coronavirus 2 (SARS-CoV-2) [1-7]. The pandemic has resulted in 3.89 million deaths worldwide, with 180 million confirmed cases thus far [8]. As in many other diseases, early detection of COVID-19 helps to provide timely treatment and save one's life. The real-time reverse transcription polymerase chain reaction (RT-PCR) test is widely used to diagnose COVID-19 [9]. However, it can achieve erroneous results and has relatively long turnaround times. The test is also costly and deep nasal swabs can be uncomfortable for some people, especially small children. Therefore, relying only on the RT-PCR test may be inadequate for the diagnosis of COVID-19 under time-sensitive situations [10]. Clinical symptoms, laboratory findings, and radiological imaging techniques such as chest computed tomography (CT) or chest radiography (X-ray) images can also be used for screening. In particular, radiological imaging techniques play a significant role in the diagnosis of COVID-19 [11,12]. The diagnosis of COVID-19 is facilitated by the bilateral patchy shadows and ground-glass opacity obtained from these techniques [13,14]. Among these techniques, a chest X-ray is faster and cheaper than CT. It also causes lower-dose radiation on the patient compared to $\mathrm{CT}$. Radiologists use these techniques to analyse images and help to diagnose COVID-19 [15,16].

Machine learning is a powerful technique used for automatic feature extraction [17-20]. Many machine learning techniques have been presented in the literature to detect different diseases [21-25]. Machine learning techniques developed especially for the early diagnosis of COVID-19 have achieved successful results [26,27]. For example, deep-learning-based methods from machine learning techniques are widely used for COVID-19 detection [28,29]. Deep-learning-based methods achieved high accuracy rates when sufficient labelled data is provided. Thus, deep-learning-based automatic diagnosis systems are of great interest in cases with no or few radiologists available [2]. Such an approach can also serve as an adjunct tool to be used by clinicians to confirm their findings.

\subsection{Motivation and Our Method}

COVID-19 is an infectious disease that has a relatively high infectivity rate. Many machine learning and signal/image processing methods have been employed to detect COVID-19 automatically using medical images or cough sounds. A new strategy is developed here to detect COVID-19 disease accurately using a novel Exemplar COVID-19FclNet9 model via both deep learning and feature engineering.

In this work, transfer learning is employed to generate hybrid deep features. It can be noted from feature engineering that exemplar feature generation is an effective method to extract discriminative features from an image. Exemplar feature extraction is processed using three pre-trained networks, namely AlexNet [30], VGG16 [31] and VGG19 [31]. These three networks are used as feature generators because each network has three fully connected layers. Using these fully connected layers, nine exemplar feature generation algorithms are then presented. The loss values of the generated features are calculated, and the top three feature vectors are selected to create a merged feature vector. The final feature vector is selected using an iterative selector; this selector is named iterative neighbourhood component analysis (INCA) [32], and the chosen feature vector is classified using a support vector machine (SVM) classifier [33,34]. Four different X-ray image databases have been utilised as testbeds to demonstrate the robustness of the presented model. We have utilised the advantages of the AlexNet, VGG16 and VGG19 CNNs together. The important features of the proposed Exemplar COVID-19FclNet9 are given below:

- choosing the most informative features automatically and 
- denoting the effectiveness of this model using a conventional classifier. Bayesian optimisation is used to tune parameters of SVM classifier.

\subsection{Literature Review}

Different COVID-19 detection models have been presented in the literature. Narin et al. [35] proposed a COVID-19 detection model. This model was based on deep convolutional neural networks (Inception-ResNetV2, InceptionV3, ResNet50-101-152). ResNet50 was most accurate in their study compared to the other pre-trained models using an X-ray image dataset. Three different databases were used to automate the model. Database1 contained data from 341 COVID-19 and 2800 normal cases. Database 2 consisted of data regarding 341 COVID-19 and 1493 viral pneumonia cases, and finally, database 3 had the data of 341 COVID-19 and 2772 bacterial images. Five-fold cross-validation was implemented to develop the model. In their study, accuracy rates of $96.10 \%, 99.50 \%$ and $99.70 \%$ were obtained using database1, database 2 and database3, respectively. Muhammad and Hossain [36] presented a COVID-19 classification method using a convolutional neural network (CNN) with lung ultrasound images. They considered three classes in their work: COVID-19, pneumonia and healthy. They reported an accuracy rate of $92.50 \%$ with their proposed method. Loey et al. [37] applied a network based on CNN and generative adversarial networks. They used a database consisting of 307 chest X-ray images with 4 classes: COVID-19, viral pneumonia, bacterial pneumonia and normal, to develop the automated model. They achieved an accuracy of $80.60 \%$ with GoogleNet with four classes. Furthermore, they attained an accuracy rate of 100.0\% for two classes (COVID-19 and normal). Their method did not achieve a high accuracy rate for four classes. Saad et al. [38] used GoogleNet, ResNet18 and deep feature concatenation for COVID-19 detection using CT and X-ray images. The data consisted of two classes: 2628 COVID-19 images and 1620 non-COVID-19 images. They achieved an accuracy of $96.13 \%$. Moreover, with deep feature concatenation, accuracy rates were $98.90 \%$ and $99.30 \%$ with CT and X-ray databases, respectively. A high accuracy rate was obtained with this method for the two-class database. Tuncer et al. [39] proposed a COVID-19 detection method using a residual exemplar local binary pattern called ResExLBP. For this, images from 87 COVID-19 and 234 healthy patients were used in their study. They reported accuracy of $100.0 \%$ with SVM classifier. The main limitation of their method is that they used a small database to develop the model. Sharma and Dyreson [40] used a residual attention network for COVID-19 detection using chest $\mathrm{X}$-ray images. In their study, 239 chest $\mathrm{X}$-ray images were utilised. Their proposed method was compared with different $\mathrm{CNN}$ models. Their method attained an accuracy of $98.00 \%$. Jia et al. [41] presented an approach based on CNN using CT and X-ray images. The modified MobileNet was used to classify five classes (COVID-19, tuberculosis, viral pneumonia, bacterial pneumonia and healthy). They obtained an accuracy of $98.80 \%$ for five-class classification. Bassi and Attux [42] proposed a deep CNN model to classify three classes (COVID-19, pneumonia and healthy) with 150 images. They reported an accuracy of $100.0 \%$. Most of the works reported above have used smaller databases, or their proposed models are computationally intensive.

\subsection{Contributions}

The novelty of the Exemplar COVID-19FclNet9 model is in the presented exemplar deep feature extractor. The feature extraction model is designed as a machine learning model. The proposed fully connected layer-based feature generator contains deep exemplar feature extraction, feature selection based on NCA [43], misclassification rate calculation with SVM, feature vector selection using the calculated misclassification rates and concatenation steps. In this work, we present a machine learning model for feature generation, and this generator aims to use deep features with maximum effectiveness. The major contributions of the proposed Exemplar COVID-19FclNet9 are given below:

- This work presents a new X-ray image classification model using deep exemplar features. This model uses three cognitive phases, as described in Section 1.1. The 
proposed model is inspired by a Vision Transformer (ViT) [44]. In addition, this work presents a lightweight and highly accurate model using three pre-trained CNNs [30]. The proposed Exemplar COVID-19FclNet9 uses cognitive feature extraction, iterative feature selection and parameters to tune the SVM classifier to achieve high classification performance.

- Many machine learning models have been presented to classify COVID-19 [7,26,45]. The proposed Exemplar COVID-19FclNet9 model has been tested using four X-ray image databases. The universal high classification ability of the Exemplar COVID$19 \mathrm{FclNet} 9$ is used to justify the robustness of the developed model.

\section{Materials and Methods}

Details of four X-ray image databases (DB1, DB2, DB3 and DB4) used in this work are given in this section.

\subsection{Materials}

\subsubsection{The First Database (DB1)}

The first database (DB1) used in this work consisted of $741 \mathrm{X}$-ray images with four classes (control/healthy, bacterial pneumonia, viral pneumonia and COVID-19). This database is a hybrid database in which normal and pneumonia images were taken from test images of Kermany et al.'s database [46,47]. COVID-19 images were taken from Talo's database [48,49]. In DB1, we have used 234 normal, 242 bacterial pneumonia, 148 viral pneumonia, and 125 COVID-19 X-ray images. Typical images are shown in Figure 1.

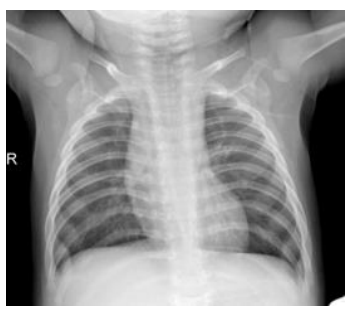

(a) Normal

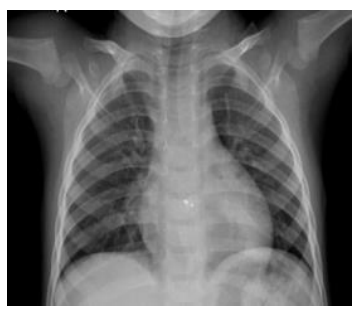

(b) Bacterial pneumonias

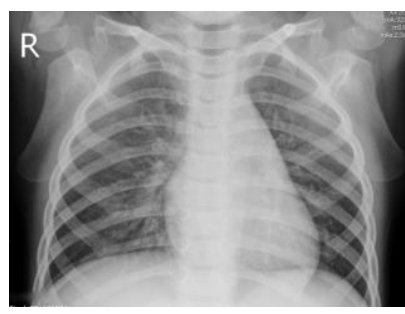

(c) Viral pneumonias

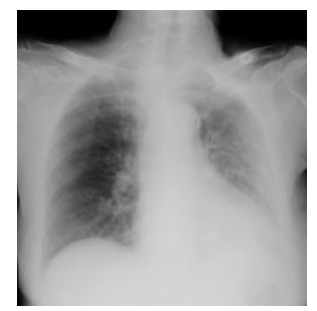

(d) COVID-19

Figure 1. Sample of images from DB1.

\subsubsection{The Second Database (DB2)}

This database is very popular [50] and was utilised to compare our results. Ozturk et al. [48] designed a novel machine learning model to detect COVID-19 and published their database and model in [49]. This database was collected from 125 subjects (43 females and 82 males). This database consists of three classes: COVID-19, pneumonia and control. The DB2 database contains 1125 (500 pneumonia, 500 control and 125 COVID-19) X-ray images. Typical images are shown in Figure 2.

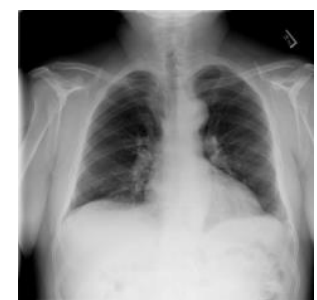

(a) COVID-19

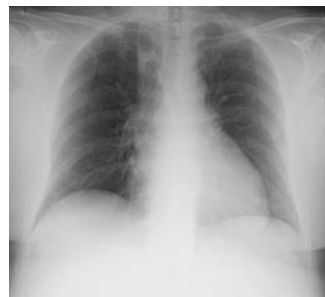

(b) Healthy

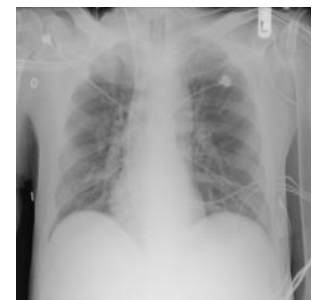

(c) Pneumonia

Figure 2. Sample of images from DB2.

\subsubsection{The Third Database (DB3)}

This dataset is a large X-ray image dataset published by Rahman in Kaggle [51], which contains three classes: no-finding, pneumonia and COVID-19 [52,53]. We used 8961 X-ray 
images (3616 COVID-19, 1345 pneumonia and 4000 normal). Typical images are shown in Figure 3.

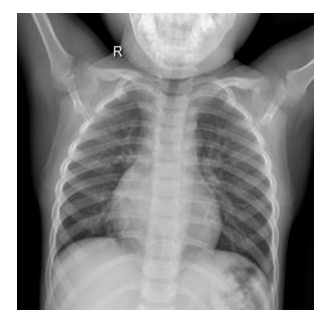

(a) COVID-19

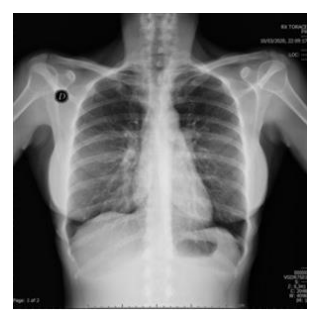

(b) Pneumonia

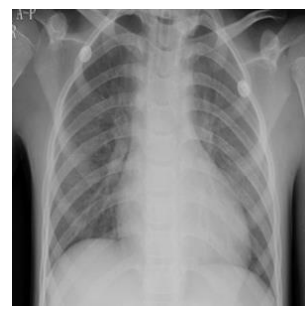

(c) Healthy

Figure 3. Sample of images from DB3.

\subsubsection{The Fourth Database (DB4)}

This database was collected from the University of Malaya Medical Centre. In all, 277 X-ray images were collected from 214 subjects. This database consists of two categories, i.e., images from 127 COVID-19 and 150 healthy patients. Typical images are shown in Figure 4.

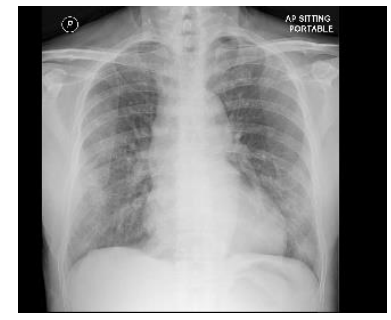

(a) COVID-19

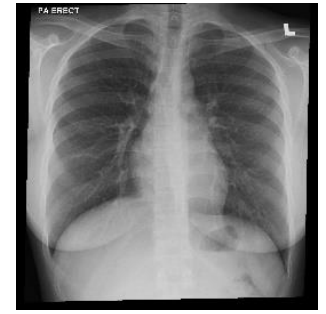

(b) Healthy

Figure 4. Sample of images from DB4.

\subsection{Methods}

In this work, the Vision Transformer (ViT) [44] design is followed, and we modified this structure to propose our model using transfer learning. This research presents a hybrid model (it uses three pre-trained deep feature generators together) to achieve maximum classification ability, and it is named Exemplar COVID-19FclNet9. ViT inspires the proposed Exemplar COVID-19FclNet9, and it uses three pre-trained deep feature generators instead of attention transformers. The schematic overview of the proposed Exemplar COVID-19FclNet9 is denoted in Figure 5.

Figure 5 summarises the presented model. The pseudocode of the proposed Exemplar COVID-19FclNet9 X-ray classification model is given in Algorithm 1.

More details about the proposed Exemplar COVID-19FclNet9 are given below.

\subsubsection{Deep Feature Extraction}

Lines 01-16 of Algorithm 1 define the presented deep feature generator. In the first stage, exemplar division has been applied to the X-ray images. In this work, the X-ray images are divided into $3 \times 3=9$ exemplars. Then, in the deep feature generator, nine deep feature extractors (three fully connected layers of three pre-trained CNNs) have been applied to the obtained nine exemplars. Finally, the original X-ray images and the extracted features are merged. The schematic explanation of the presented deep feature generator is shown in Figure 6. 


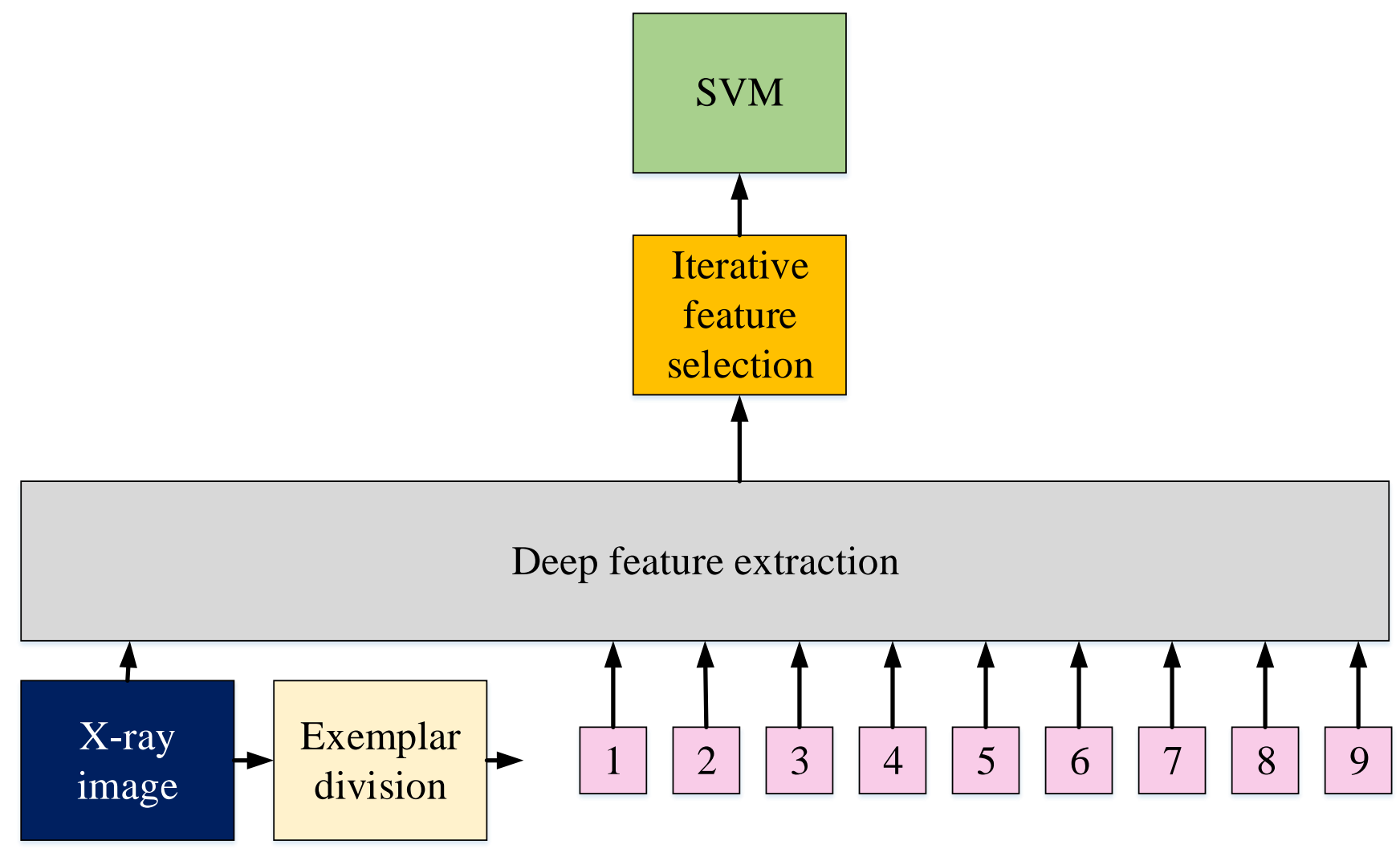

Figure 5. Graphical illustration of proposed Exemplar COVID-19FclNet9 model.

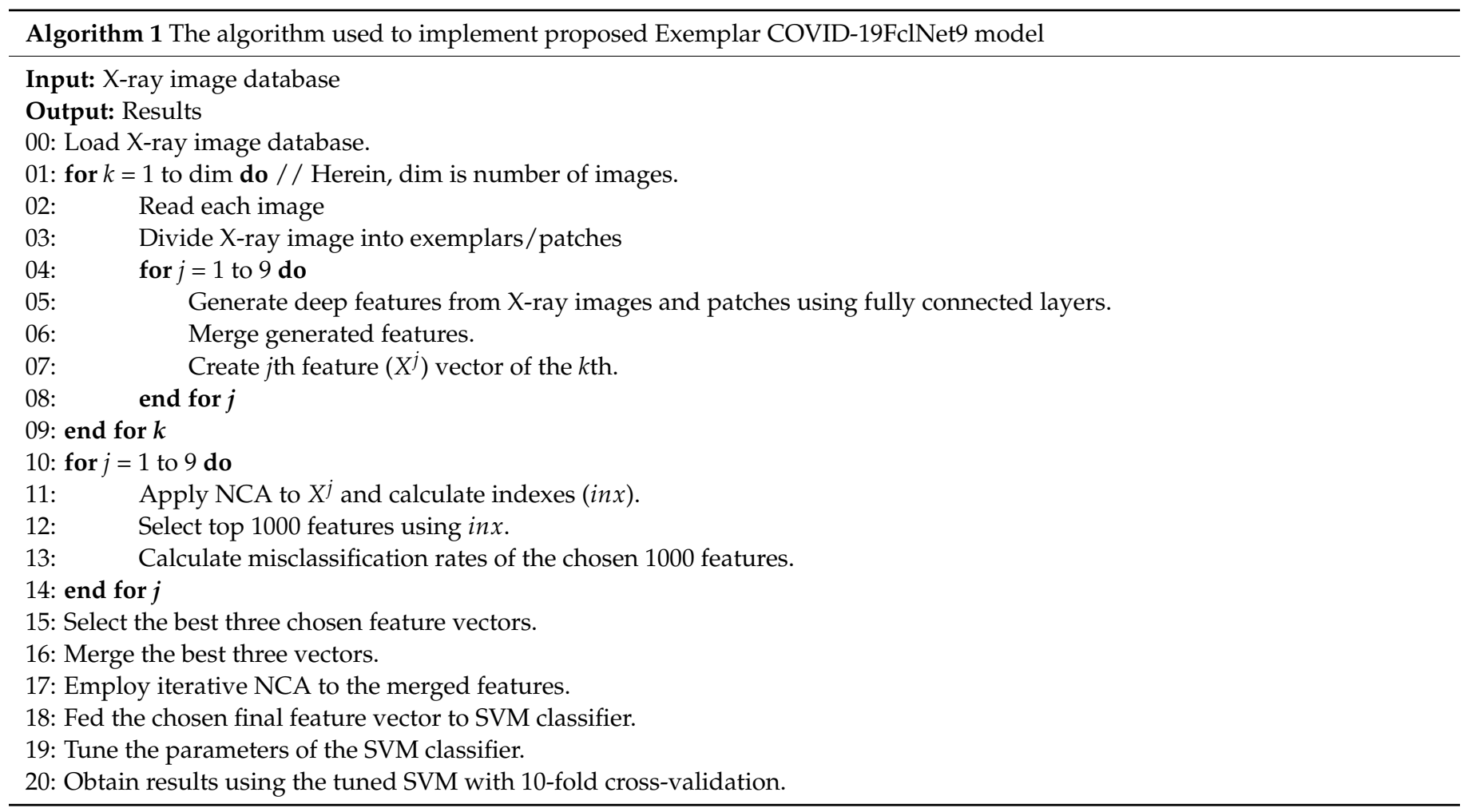




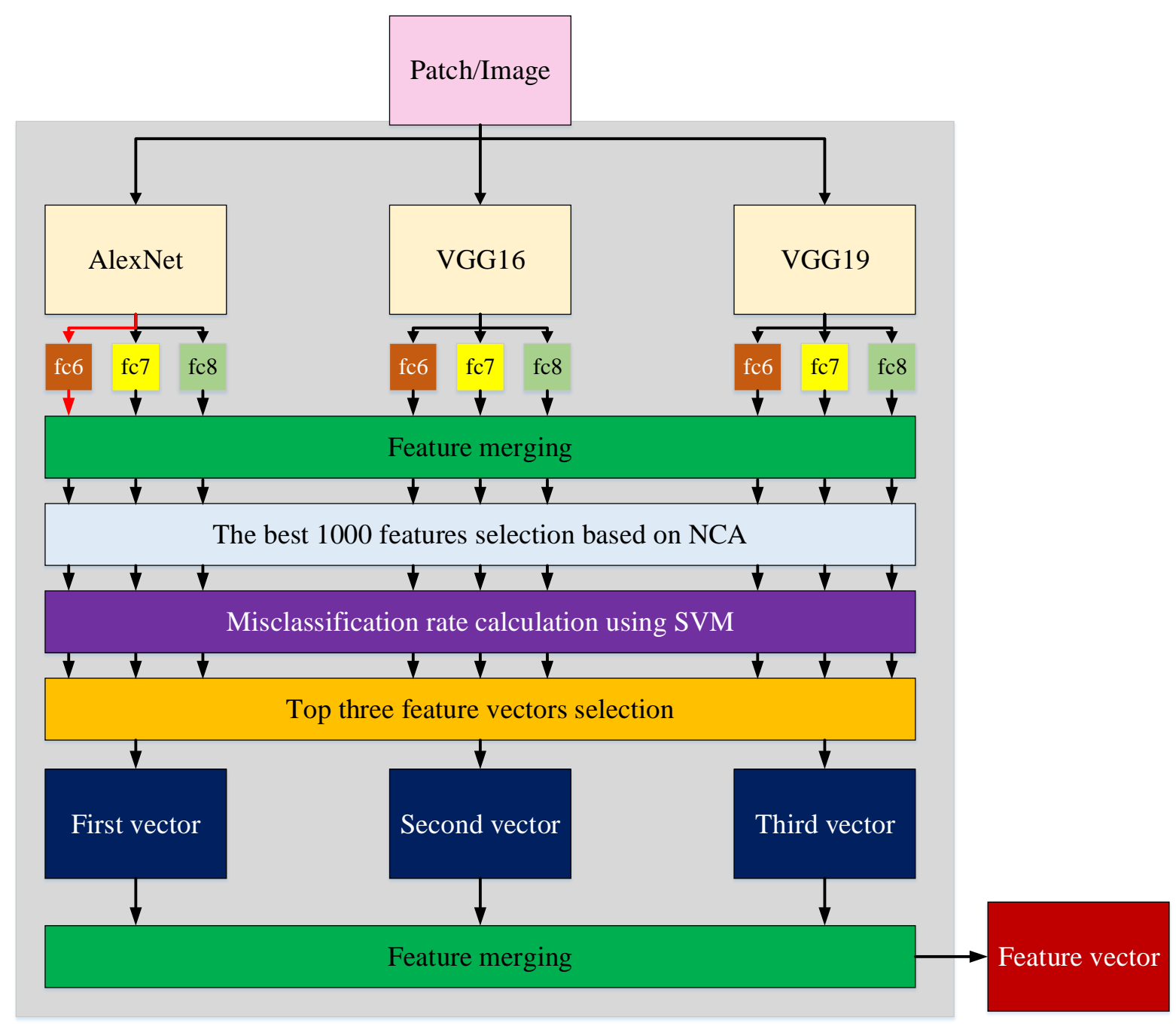

Figure 6. Detailed representation of proposed deep feature generator. The red arrow shows the activated feature extraction cell. All fully connected layers are activated consecutively in the presented hybrid deep feature generator.

Steps of the presented deep feature generator are given below:

Step 1: Create non-overlapping patches.

$$
\begin{gathered}
p t^{c n t}(p, r, k)=I(i: i+p-1, j: j+r-1, k), c n t \in\{1,2, \ldots, 9\} \\
p \in\left\{1,2, \ldots,\left\lfloor\frac{w}{3}\right\rfloor\right\}, r \in\left\{1,2, \ldots,\left\lfloor\frac{h}{3}\right\rfloor\right\}, i \in\left\{1,\left\lfloor\frac{w}{3}\right\rfloor, \ldots, w\right\}, j \in\left\{1,\left\lfloor\frac{h}{3}\right\rfloor, \ldots, h\right\}
\end{gathered}
$$

Equations (1) and (2) define patch creating. In this work, nine patches are created, where $p t^{c n t}$ is $c n t^{t h}$ patch and $c n t$ is a counter for the patches, I represents the original $\mathrm{X}$-ray image, $w$ denotes the width of the used X-ray image, $h$ is the height of the used X-ray image and $i, j, k, p, r$ are indices.

Step 2: Extract nine features from $X$-ray images and patches using nine fully connected layers.

$$
\begin{gathered}
X^{q}(h, 1: s z)=\operatorname{fem}^{q}(I), q \in\{1,2, \ldots, 9\}, h \in\{1,2, \ldots, \operatorname{dim}\} \\
X^{q}(h, q \times s z+1:(q+1) \times s z)=f e m^{q}\left(p t^{c n t}\right)
\end{gathered}
$$

In Equations (3) and (4), $X^{q}$ is $q$ th feature vector, $\mathrm{fem}^{q}$ is qth feature extraction method. In this work, fc6, fc7 and fc8 layers of the AlexNet, VGG16 and VGG19 were used to 
generate deep features, $s z$ denotes the length of the generated feature vector. The fc8 layer of the used networks extracts 1000 features (sz is 1000 for $\mathrm{fem}^{3}, \mathrm{fem}^{6}, \mathrm{fem}^{9}$ ). Moreover, 4096 features are generated using the fc6 and fc7 layers of all used CNNs (sz is 4096 for $\mathrm{fem}^{1}, \mathrm{fem}^{2}, \mathrm{fem}^{4}, \mathrm{fem}^{5}, \mathrm{fem}^{7}, \mathrm{fem}^{8}$ ). The presented deep extractor creates features from $\mathrm{X}$-ray images and nine patches. The Equations (3) and (4) define both feature generation and merging. The length of the generated $X^{3}, X^{6}, X^{9}$ is 10,000 and the length of the other feature vector is 40,960 .

Step 3: Select the best 1000 features from each generated feature vector deploying NCA.

$$
\begin{gathered}
\text { inx } x^{q}=\operatorname{NCA}\left(X^{q}, y\right) \\
f c^{q}(h, j)=X^{q}\left(h, i n x^{q}(j)\right), j \in\{1,2, \ldots, 1000\}
\end{gathered}
$$

where in $x^{q}$ are the qualified indexes of the qth feature vector, $y$ represents actual output, $N C A(.,$.$) defines NCA feature selection function and f c^{q}$ is qth chosen feature vector with a length of 1000 .

In this step, 1000 features are selected from generated feature vectors as in many pre-trained CNNs.

Step 4: Calculate misclassification rates of the $f c$ using polynomial kernelled SVM with 10 -fold cross-validation.

$$
\operatorname{loss}(q)=\operatorname{SVM}\left(f c^{q}\right)
$$

Herein, loss is the misclassification rate of the selected features.

Step 5: Select the best three selected feature vectors using calculated loss values in Step 4.

$$
\begin{gathered}
{[q l, i d]=\operatorname{sort}(\text { loss })} \\
t f^{i}=f c^{i d(i)}, i \in\{1,2,3\}
\end{gathered}
$$

Herein, $q l$ is qualified loss by ascending, sort (.) defines the sorting function, $i d$ represents sorted indexes and $t f^{1}, t f^{2}, t f^{3}$ are the top three feature vectors.

Step 6: Merge the selected three feature vectors and obtain generated features.

$$
X^{G}=t f^{1}\left|t f^{2}\right| t f^{3}
$$

Herein, $X^{G}$ is the best-merged feature with a length of 3000 and | is the merging operator.

\subsubsection{Iterative Feature Selector}

In order to choose the best features from the generated $X^{G}$, the iterative NCA (INCA) [32] feature selector was employed. INCA is an iterative and improved version of the NCA that helps to select the most appropriate feature vector with optimal length. It is a parametric feature selector, and the parameters of this selector are loss function and range of iteration (initial and end values). In this work, the SVM classifier was used as a loss function, and $[100,1000]$ was selected as the range. INCA can select different-sized feature vectors for different problems. The steps of the INCA are given below.

Step 7: Generates qualified indexes of the features.

Step 8: Select 901 features using generated qualified indexes. The lengths of the first and last feature vectors are 100 and 1000.

Step 9: Calculate loss values of each feature vector using SVM, which is equal to 1-accuracy (i.e., 901 in this case). The computed errors are shown in Figure 7.

Step 10: Find the index of minimum error and select the best feature vector from the index (see Figure 7).

We applied INCA to generate the feature vector and obtain the best features for classification. INCA selected 340, 509, 735 and 101 features for DB1, DB2, DB3 and DB4 databases, respectively. The plots of misclassification rate versus the number of features 
obtained for various databases using Cubic SVM with 10-fold cross-validation are shown in Figure 7.

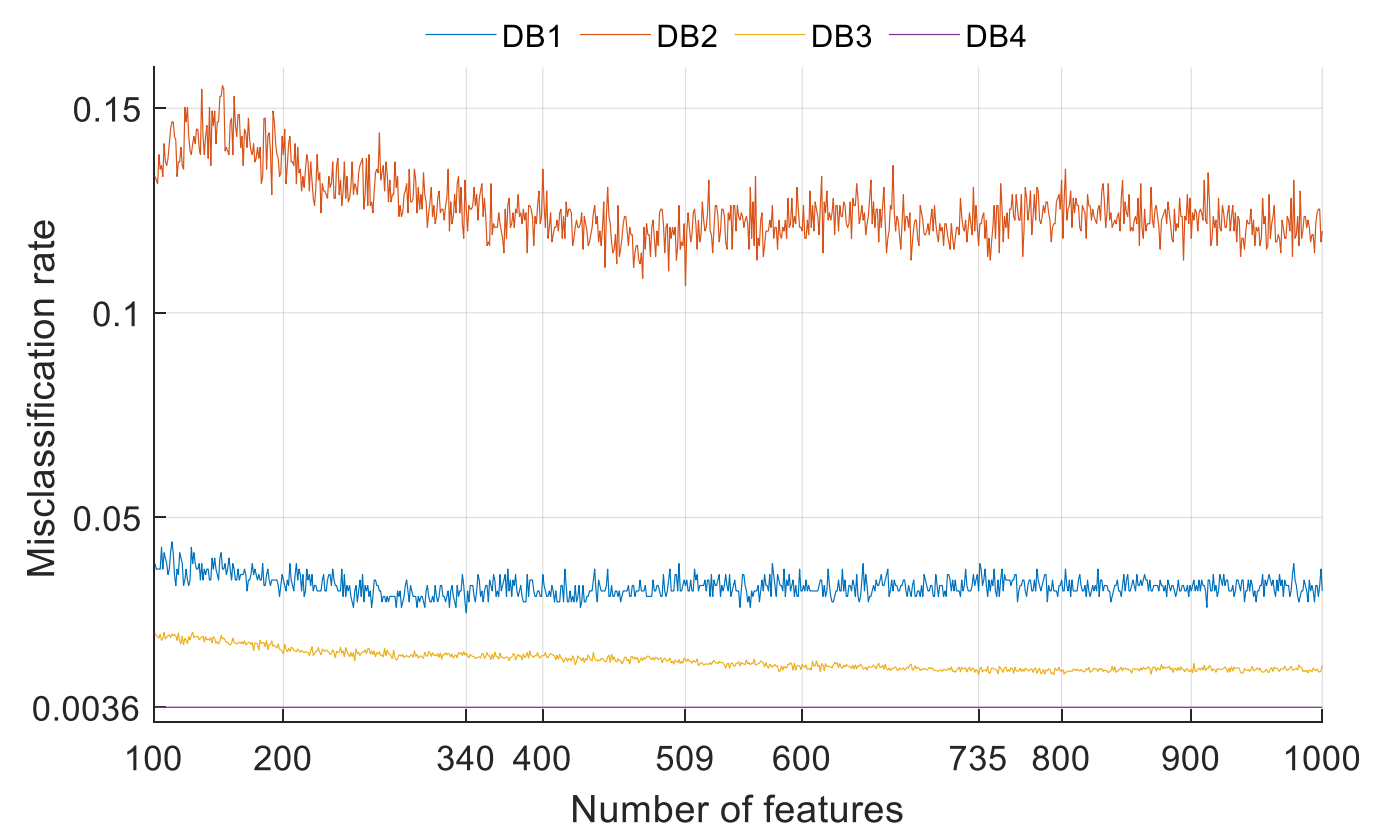

Figure 7. Plot of misclassification rate versus the number of features obtained for various databases.

\subsubsection{Classification}

The classification was performed using an SVM $[33,34]$ classifier. The hyperparameters of the SVM were tuned using Bayesian optimisation to reach the optimum performance. The number of iterations of the Bayesian optimisation was chosen to be 30 , and the fitness function was the misclassification rate. The hyperparameters of the SVM classifier (tabulated in Table 1) were fed as input to the Bayesian optimisation technique. The main purpose of using Bayesian optimisation was to obtain fine-tuned SVM, and the hyperparameter ranges used for Bayesian optimisation are given in Table 1.

Table 1. Hyperparameter ranges tuned by Bayesian optimiser for SVM classifier.

\begin{tabular}{cc}
\hline Hyperparameter & Value \\
\hline Multiclass method & One-vs.-One, One-vs.-All \\
Box constraint level & {$[0.001-1000]$} \\
Kernel & Cubic, Quadratic, Linear, Gaussian \\
Standardise & False, True \\
\hline
\end{tabular}

The parameters of the used SVM classifiers for both databases are given in Table 2.

Table 2. Hyperparameters used for SVM classifiers with various databases.

\begin{tabular}{cccc}
\hline Hyperparameter & $\begin{array}{c}\text { Tuned Parameters } \\
\text { for the DB1 }\end{array}$ & $\begin{array}{c}\text { Tuned Parameters } \\
\text { for the DB2 }\end{array}$ & $\begin{array}{c}\text { Tuned Parameters } \\
\text { for the DB3 }\end{array}$ \\
\hline Multiclass method & One-vs.-One & One-vs.-All & One-vs.-All \\
Kernel & Linear & Gaussian & Cubic \\
Box constraint & 999.30 & 2 & 1 \\
Standardise & False & True & True \\
\hline
\end{tabular}

The validation technique of the given classifiers (see Table 2) was chosen as 10-fold cross-validation.

The last step of the COVID-19FclNet9 is classification, and these steps are given below.

Step 11: Tune parameters of SVM using Bayesian optimisation.

Step 12: Classify the selected optimal feature vector using fine-tuned SVM. 


\section{Results}

This work used four X-ray image databases to validate the proposed Exemplar COVID19FclNet9 model. A simple configured PC was used to obtain the results of this model. The system configurations of the used PC are as follows. It has an i9-9900 processor, 48 GB memory, 256 GB solid-state disk, and Windows 10.1 Professional operating system. In addition, MATLAB (2020b) has been utilised as a programming environment.

To evaluate the performance of the proposed model, four databases were used. Accuracy, precision, recall and F1 score metrics were employed to evaluate the performance of the developed model. The results obtained for the four databases are listed in Tables 3-6.

Table 3. Results obtained using our proposed Exemplar COVID-19FclNet9 model with DB1 database.

\begin{tabular}{ccccc}
\hline \multirow{2}{*}{ Actual Class } & \multicolumn{3}{c}{ Predicted Class } \\
\cline { 2 - 5 } & Normal & Bacterial Pneumonia & Virus Pneumonia & COVID-19 \\
\hline Normal & 227 & 4 & 3 & 0 \\
Bacterial Pneumonia & 3 & 238 & 1 & 0 \\
Viral Pneumonia & 3 & 4 & 141 & 0 \\
COVID-19 & 0 & 9 & 0 & 125 \\
Recall (\%) & 97.01 & 98.35 & 95.27 & 100 \\
Precision (\%) & 97.42 & 96.75 & 97.24 & 100 \\
F1-score (\%) & 97.22 & 97.54 & 96.25 & \\
\hline
\end{tabular}

Table 4. Results obtained using our proposed Exemplar COVID-19FclNet9 model with DB2 database.

\begin{tabular}{cccc}
\hline \multirow{2}{*}{ Actual Class } & \multicolumn{3}{c}{ Predicted Class } \\
\cline { 2 - 4 } & COVID-19 & Healthy & Pneumonia \\
\hline COVID-19 & 120 & 0 & 5 \\
Healthy & 1 & 457 & 42 \\
Pneumonia & 0 & 65 & 432 \\
Recall (\%) & 96 & 91.40 & 87 \\
Precision (\%) & 99.17 & 87.55 & 90.25 \\
F1-score (\%) & 97.56 & 89.43 & 88.59 \\
\hline
\end{tabular}

Table 5. Results obtained using our proposed Exemplar COVID-19FclNet9 model with DB3 database.

\begin{tabular}{cccc}
\hline & \multicolumn{3}{c}{ Predicted Class } \\
\cline { 2 - 4 } Actual Class & COVID-19 & Pneumonia & Healthy \\
\hline COVID-19 & 3586 & 2 & 28 \\
Pneumonia & 2 & 1318 & 25 \\
Healthy & 28 & 19 & 3953 \\
Recall (\%) & 99.17 & 97.99 & 98.82 \\
Precision (\%) & 99.17 & 98.43 & 98.68 \\
F1-score (\%) & 99.17 & 98.21 & 98.75 \\
\hline
\end{tabular}

Table 6. Results obtained using our proposed Exemplar COVID-19FclNet9 model with DB4 database.

\begin{tabular}{ccc}
\hline & \multicolumn{2}{c}{ Predicted Class } \\
\cline { 2 - 3 } Actual Class & COVID-19 & Healthy \\
\hline COVID-19 & 126 & 1 \\
Healthy & 0 & 150 \\
Recall (\%) & 99.21 & 100 \\
Precision (\%) & 100 & 99.33 \\
F1-score (\%) & 99.60 & 99.66 \\
\hline
\end{tabular}

The biggest database used was DB3, and its results were obtained with 10-fold crossvalidation. The calculated confusion matrix of our proposed Exemplar COVID-19FclNet9 model using the DB3 database is denoted Table 5. 
The overall results (accuracy, unweighted average recall, overall precision and overall F1 scores) obtained using our proposed model with four databases is shown in Table 7.

Table 7. Overall results (\%) obtained using our proposed model using four databases.

\begin{tabular}{ccccc}
\hline Overall Results & DB1 & DB2 & DB3 & DB4 \\
\hline Accuracy (\%) & 97.60 & 89.96 & 98.84 & 99.64 \\
Unweighted average recall (\%) & 97.66 & 91.47 & 98.66 & 99.61 \\
Precision (\%) & 97.85 & 92.32 & 98.76 & 99.80 \\
F1 score (\%) & 97.75 & 91.86 & 98.71 & 99.63 \\
\hline
\end{tabular}

It can be noted from Table 7 that our proposed model obtained $97.60 \%, 89.96 \%, 98.84 \%$ and $99.64 \%$ accuracies using DB1, DB2, DB3 and DB4 databases, respectively.

Moreover, ROC curves obtained using our proposed COVID-19FclNet9 model for various datasets used are denoted in Figure 8.

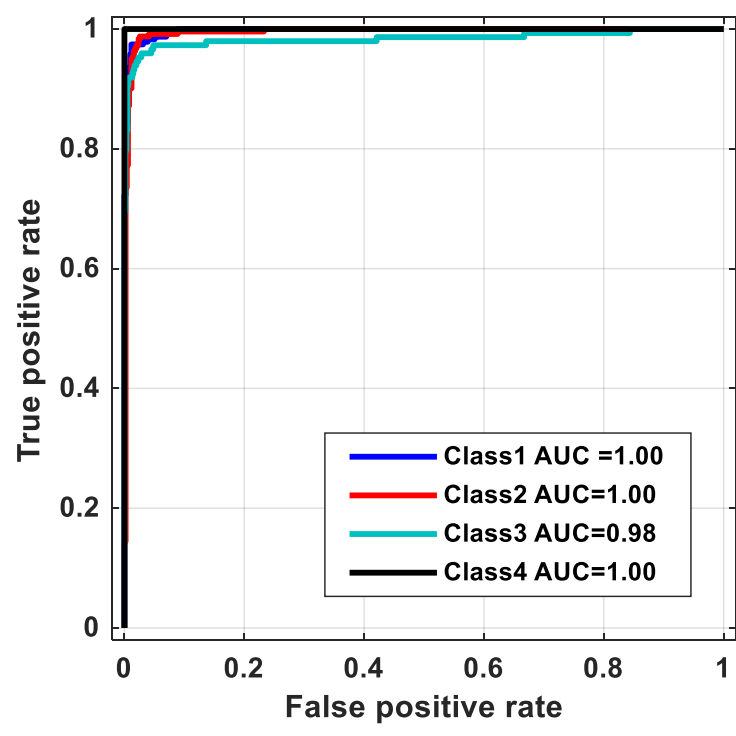

(a)

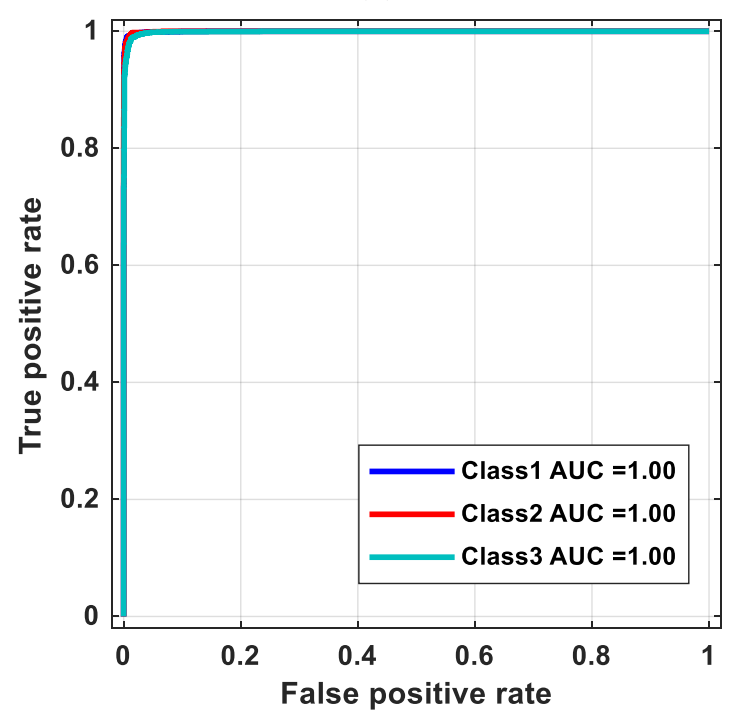

(c)

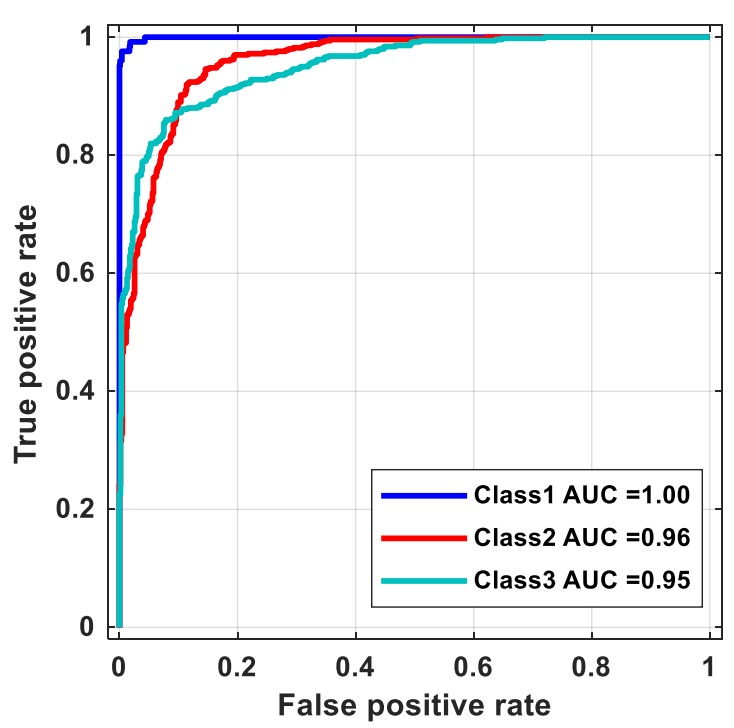

(b)

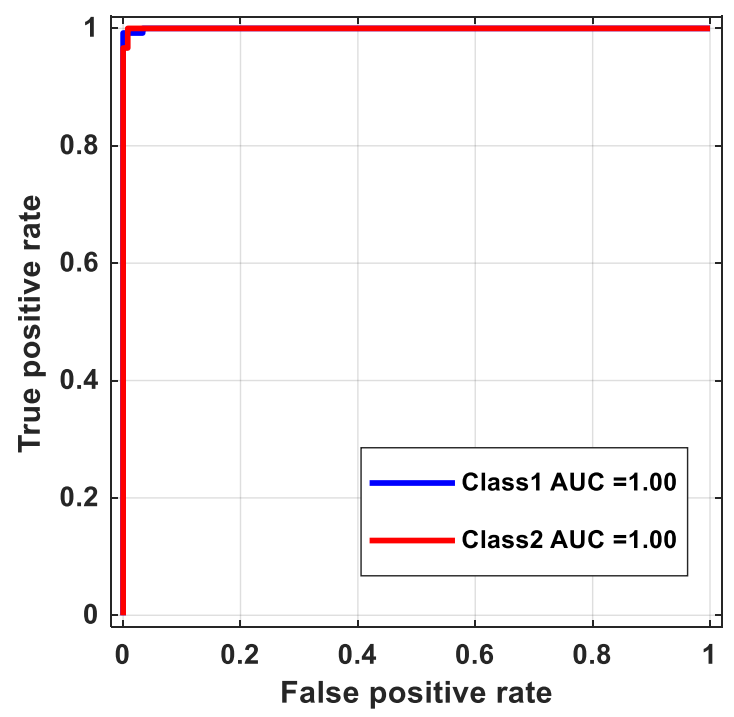

(d)

Figure 8. ROC curves of proposed model for datasets used: (a) DB1, (b) DB2, (c) DB3 and (d) DB4. 


\section{Discussion}

Four X-ray image corpora were used in this work to validate the proposed Exemplar COVID-19FclNet9 model. It can be noted from our results that the developed model yielded high classification performance using all four databases. The proposed model is cognitive, and we used feature engineering and transfer learning to design this architecture. This model has three fundamental phases, and the most crucial phase of the proposed model is feature extraction. Our proposed deep feature generation model is a cognitive model and is also designed as a machine learning model. This feature extractor selects the most appropriate three feature vectors. It generates features using patches and original X-ray images, and these features are merged. NCA chooses the best 1000 features, which are classified using SVM classifier. The used pre-trained deep feature generation models are listed in Table 8.

Table 8. Deep feature generation functions used in the Exemplar COVID-19FclNet9 model.

\begin{tabular}{ccc}
\hline Network & Number & Fully Connected Layer \\
\hline \multirow{3}{*}{ AlexNet } & 1 & $\mathrm{fc} 8$ \\
& 2 & $\mathrm{fc7}$ \\
& 3 & $\mathrm{fc6}$ \\
\hline \multirow{2}{*}{ VGG16 } & 4 & $\mathrm{fc} 8$ \\
& 5 & $\mathrm{fc7}$ \\
& 6 & $\mathrm{fc6}$ \\
\hline \multirow{2}{*}{ VGG19 } & 7 & $\mathrm{fc} 8$ \\
& 8 & $\mathrm{fc7}$ \\
& 9 & $\mathrm{fc6}$ \\
\hline
\end{tabular}

Table 8 shows the deep feature generation functions used in the Exemplar COVID$19 F c 1 N e t 9$ model. The graph of accuracies versus number of features used with various databases is depicted in Figure 9.

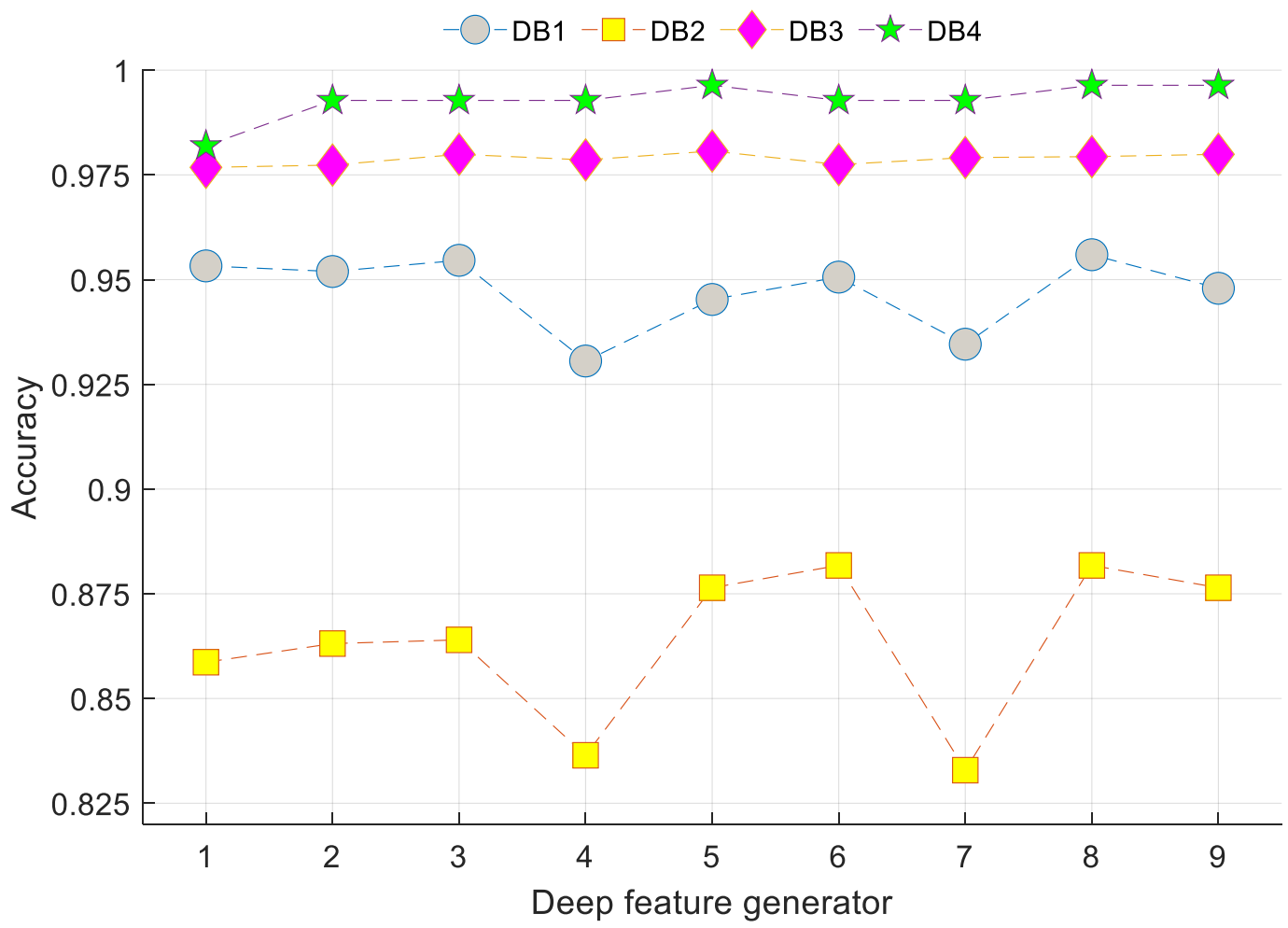

Figure 9. Graph of accuracies versus number of features used for various datasets used. 
Figure 9 shows that the range of accuracies calculated for DB1 varies from $93.06 \%$ (minimum accuracy) to $95.59 \%$ (maximum accuracy), and this range can be expressed as [93.06\%, 95.59\%]. Moreover, the obtained accuracy ranges for DB2, DB3 and DB4 were [83.29\%, 88.18\%], [97.68\%, 98.07\%] and [98.19\%, 99.64\%], respectively, using nine deep feature generation methods. The best three feature generators used for DB1 were the 8th (fc7 layer of the VGG19), 3rd (fc6 layer of the AlexNet) and 1st (fc8 layer of the AlexNet) deep feature generators. The selected three deep features for DB2 belonged to 6th (fc6 layer of the VGG16), 8th (fc7 layer of the VGG19) and 5th (fc7 layer of the VGG16) deep feature generators. The top three feature generators for DB3 were the 5th (fc7 layer of the VGG16), 3rd (fc6 layer of the AlexNet) and 9th (fc6 layer of the VGG19) transfer learning-based deep feature generators. Furthermore, 5th (fc7 layer of the VGG16), 8th (fc7 layer of the VGG19) and 9th (fc6 layer of the VGG19) were selected as the top three deep feature generators.

By merging these features and applying the INCA selector, the accuracy rates were increased from $95.59 \%$ to $97.60 \%$ for DB1, from $88.18 \%$ to $89.96 \%$ for DB2 and from $98.07 \%$ to $98.84 \%$ for DB3. Moreover, this strategy yielded the maximum performance with the minimum number of features (100 features) on the DB4.

The comparison of our work with other similar published works is shown in Table 9.

It can be noted from Table 9 that our proposed method has outperformed all the state-of-the-art techniques and is found to be robust as we have tested with four different databases. We used four X-ray image datasets to evaluate the presented COVID-19FclNet9. We used both small and large datasets. Murugan and Goel [54] applied a CNN to classify COVID-19, pneumonia and healthy classes. Their used dataset contained 2700 images, and each category had 900 images. Gilanie et al. [55] used a large X-ray image dataset, and their image dataset contained 15,108 X-ray images. They only applied VGG-16 network and reached $96 \%$ classification accuracy.

Ozturk et al. [48] proposed a convolutional neural network using an X-ray image dataset of three classes (COVID-19, pneumonia and healthy). Furthermore, their dataset is a heterogeneous dataset and was the DB2 dataset in our work. Ozturk et al. [48] achieved $87.02 \%$ accuracy using their model, while our COVID-19FclNet9 reached $89.96 \%$ accuracy using the same dataset. Hussain et al. [58] presented a CNN-based CoroDet model using three datasets and attained $99.10 \%, 94.20 \%$ and $91.20 \%$ classification accuracies, respectively. They did not use transfer learning; hence, the time complexity of their model may be higher than that for our proposal. Sitaula and Hossain [61] used transfer-learning-based deep X-ray image classification and used three image datasets. They reached 79.58\%, 85.43\% and $87.49 \%$ accuracies for three image datasets, respectively. Our COVID-19FclNet9 is a transfer-learning-based model, and it attained higher accuracies than that of the Sitaula and Hossain [61] transfer-learning-based classification model. Other methods in Table 5 used a $\mathrm{CNN}$ model with smaller X-ray image datasets. In this respect, our work is one of the first to use four datasets, as shown in Table 9, and obtained a higher classification performance for X-ray image classification. This justifies that our proposed model is accurate and robust. 
Table 9. Comparison of our work with other similar published works.

\begin{tabular}{|c|c|c|c|c|c|c|}
\hline Study & Method & Classifier & Split Ratio & Number of Class/Type & Number of Cases & Results (\%) \\
\hline Murugan and Goel [54] & $\begin{array}{c}\text { Convolutional neural } \\
\text { networks } \\
\text { (ResNet50) }\end{array}$ & Softmax & $70: 30$ & 3/Chest X-ray & $\begin{array}{l}900 \text { COVID-19 } \\
900 \text { Pneumonia } \\
900 \text { Normal }\end{array}$ & $\begin{array}{c}\text { Acc: } 94.07 \\
\text { Sen: } 98.15 \\
\text { Spe: } 91.48 \\
\text { Rec: } 85.21 \\
\text { Pre: } 98.15 \\
\text { F1: } 91.22\end{array}$ \\
\hline Gilanie et al. [55] & $\begin{array}{c}\text { Convolutional neural } \\
\text { networks }\end{array}$ & Softmax & $60: 20: 20$ & Chest radiology & $\begin{array}{c}1066 \text { COVID-19 } \\
7021 \text { Pneumonia } \\
7021 \text { Normal }\end{array}$ & $\begin{array}{l}\text { Acc: } 96.68 \\
\text { Spe: } 95.65 \\
\text { Sen: } 96.24 \\
\end{array}$ \\
\hline Pandit et al. [56] & $\begin{array}{c}\text { Convolutional neural } \\
\text { networks } \\
\text { (VGG-16) }\end{array}$ & Softmax & $70: 30$ & $\begin{array}{l}\text { 1. } 2 \text { /Chest radiographs } \\
\text { 2. } 3 / \text { Chest radiographs }\end{array}$ & $\begin{array}{c}1 . \\
224 \text { COVID-19 } \\
504 \text { Healthy } \\
2 . \\
224 \text { COVID-19 } \\
700 \text { Pneumonia } \\
504 \text { Healthy }\end{array}$ & $\begin{array}{c}\text { Acc: } \\
\text { 1. } 96.00 \\
\text { 2. } 92.53\end{array}$ \\
\hline Nigam et al. [57] & $\begin{array}{c}\text { Convolutional neural } \\
\text { networks } \\
\text { (EfficientNet) }\end{array}$ & Softmax & 70:20:10 & 3/Chest X-ray & $\begin{array}{l}795 \text { COVID-19 } \\
795 \text { Normal } \\
711 \text { Others }\end{array}$ & Acc: 93.48 \\
\hline Hussain et al. [58] & $\begin{array}{c}\text { Convolutional neural } \\
\text { networks } \\
\text { (CoroDet) }\end{array}$ & Softmax & $\begin{array}{l}\text { 5-fold cross } \\
\text { validation }\end{array}$ & $\begin{array}{l}\text { 1. 2/Chest X-ray } \\
\text { 2. 3/Chest X-ray } \\
\text { 3. 4/Chest X-ray }\end{array}$ & $\begin{array}{c}1 . \\
500 \text { COVID-19 } \\
800 \text { Normal } \\
2 . \\
500 \text { COVID-19 } \\
800 \text { Normal } \\
800 \text { Pneumonia-bacterial } \\
3 . \\
500 \text { COVID-19 } \\
800 \text { Normal } \\
400 \text { Pneumonia-bacterial } \\
400 \text { Pneumonia-viral }\end{array}$ & $\begin{array}{c}\text { Acc: } \\
\text { 1. } 99.10 \\
\text { 2. } 94.20 \\
\text { 3. } 91.20\end{array}$ \\
\hline
\end{tabular}


Table 9. Cont.

\begin{tabular}{|c|c|c|c|c|c|c|}
\hline Study & Method & Classifier & Split Ratio & Number of Class/Type & Number of Cases & Results (\%) \\
\hline Ozturk et al. [48] & Deep neural networks & Darknet-19 & $\begin{array}{l}\text { 5-fold cross } \\
\text { validation }\end{array}$ & 3/Chest X-ray & $\begin{array}{l}125 \text { COVID-19 } \\
500 \text { Pneumonia } \\
500 \text { No Findings }\end{array}$ & $\begin{array}{l}\text { Acc: } 87.02 \\
\text { Sen: } 92.18 \\
\text { Spe: } 89.96\end{array}$ \\
\hline Shi et al. [59] & Deep neural networks & $\begin{array}{l}\text { Deep neural } \\
\text { networks }\end{array}$ & $70: 20: 10$ & $\begin{array}{l}\text { 1. 3/Chest CT images } \\
\text { 2. 3/Chest X-ray }\end{array}$ & $\begin{array}{c}1 . \\
349 \text { COVID-19 } \\
384 \text { Normal } \\
304 \text { CAP } \\
2 . \\
450 \text { COVID-19 } \\
1800 \text { Normal } \\
1837 \text { CAP }\end{array}$ & $\begin{array}{l}\text { Acc: } \\
\text { 1. } 87.98 \\
\text { 2. } 93.44\end{array}$ \\
\hline Mukherjee et al. [60] & $\begin{array}{l}\text { Convolutional neural } \\
\text { network, Deep neural } \\
\text { network }\end{array}$ & Softmax & $\begin{array}{l}\text { 10-fold cross } \\
\text { validation }\end{array}$ & $\begin{array}{c}\text { 2/Computed } \\
\text { Tomography and Chest } \\
\text { X-ray }\end{array}$ & $\begin{array}{c}336 \text { COVID-19 } \\
336 \text { non-COVID-19 }\end{array}$ & $\begin{array}{c}\text { Acc: } 96.28 \\
\text { Sen: } 97.92 \\
\text { Spe: } 94.64 \\
\text { Pre: } 94.81 \\
\text { F1: } 96.34\end{array}$ \\
\hline Sitaula and Hossain [61] & $\begin{array}{c}\text { Convolutional neural } \\
\text { networks }\end{array}$ & $\begin{array}{l}\text { FC-layers, and } \\
\text { Softmax }\end{array}$ & $70: 30$ & $\begin{array}{l}\text { 1. 3/Chest X-ray } \\
\text { 2. 4/Chest X-ray } \\
\text { 3. } 5 / \text { Chest X-ray }\end{array}$ & $\begin{array}{c}\text { Database1: } \\
\text { 125 COVID-19 } \\
125 \text { No findings } \\
\text { 125 Pneumonia } \\
\text { Database2: } \\
\text { 320 COVID-19 } \\
320 \text { Normal } \\
320 \text { Pneumonia Bacterial } \\
320 \text { Pneumonia Viral } \\
\text { Database3: } \\
\text { 320 COVID-19 } \\
320 \text { Normal } \\
320 \text { Pneumonia Bacterial } \\
320 \text { Pneumonia Viral } \\
320 \text { No findings }\end{array}$ & $\begin{array}{l}\text { Acc: } \\
\text { 1. } 79.58 \\
\text { 2. } 85.43 \\
\text { 3. } 87.49\end{array}$ \\
\hline
\end{tabular}


Table 9. Cont.

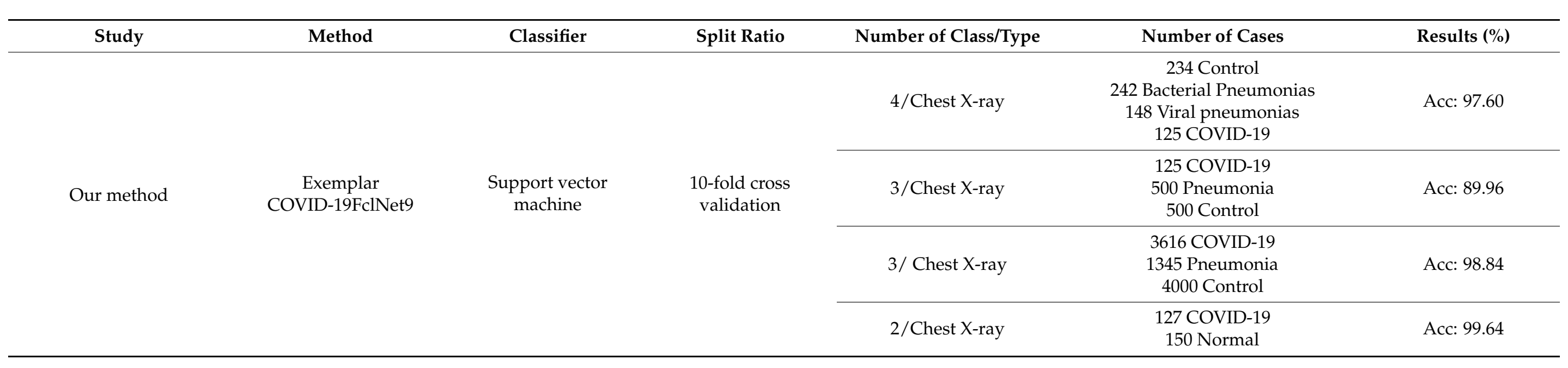



below.

The important salient features of the proposed Exemplar COVID-19FclNet9 are given

- A new deep feature generation architecture is presented using three pre-trained networks, and the proposed architecture can select the best feature generation model.

- This exemplar and cognitive deep feature generation model tested using four COVID-19 $\mathrm{X}$-ray image databases and attained a high success rate on all databases, which justifies the universal success of this model.

- This model attained $97.60 \%, 89.96 \%, 98.84 \%$ and $99.64 \%$ accuracies using four databases (DB1, DB2, DB3 and DB4, respectively).

- Our method obtained the highest performance compared to other state-of-the-art works (see Table 9).

- The proposed method is a cognitive model because it can automatically select the best models, best features and most appropriate classifier.

- The proposed model yielded the highest classification performance using deep feature generators.

- The proposed model can detect COVID-19 and pneumonia accurately using X-ray images.

\section{Conclusions}

COVID-19 detection using medical images is a topic of immense interest in medical and healthcare research. Many methods have been proposed to detect COVID-19 accurately using image processing and machine learning techniques. For example, deep networks have been applied to COVID-19 cases using X-ray images. Our work here has presented a new Exemplar COVID-19FclNet9 framework to detect COVID-19 cases automatically. In this framework, nine deep feature extraction methods are obtained using three deep networks and this framework selects the most appropriate features. Using the proposed hybrid deep feature extractor, iterative feature selector and optimised SVM, a highly accurate model is then obtained. This model was tested on several X-ray image databases to confirm the universal classification. Vision transformers inspired this learning framework (Exemplar COVID-19FclNet9) and helped increase the performance of pre-trained deep feature extractors. Our proposed Exemplar COVID-19FclNet9 attained accuracies of $97.60 \%, 89.96 \%, 98.84 \%$ and $99.64 \%$ for four X-ray image databases (DB1, DB2, DB3 and DB4, respectively). It can be noted from these results that COVID-19FclNet9 is an effective computer vision model presented using AlexNet, VGG16 and VGG19 networks. In future work, variable pre-trained deep networks can be used in this architecture to improve performance.

Author Contributions: Conceptualisation, P.D.B., N.F.M.G., K.R., N.R., W.L.N., W.Y.C., M.K., S.D., M.B., O.Y., T.T., K.H.C. and U.R.A.; formal analysis, P.D.B., N.F.M.G., K.R., N.R., W.L.N., W.Y.C., M.K., S.D., M.B., O.Y., T.T., T.W., K.H.C. and U.R.A.; investigation, S.D., M.B., O.Y. and T.T.; methodology, S.D., M.B., O.Y. and T.T.; project administration, U.R.A.; resources, P.D.B., N.F.M.G., K.R., N.R., W.L.N., W.Y.C., T.W. and K.H.C.; supervision, P.D.B., N.F.M.G., K.R., N.R., W.L.N., W.Y.C., S.D., T.T., K.H.C. and U.R.A.; validation, P.D.B., N.F.M.G., K.R., N.R., W.L.N., W.Y.C., S.D., T.T., T.W. and K.H.C.; visualisation, P.D.B., N.F.M.G., K.R., N.R., W.L.N., W.Y.C., M.K., S.D., M.B., O.Y., T.T., T.W. and K.H.C.; writing-original draft, S.D., M.B., O.Y. and T.T.; writing-review and editing, P.D.B., N.F.M.G., K.R., N.R., W.L.N., W.Y.C., M.K., S.D., M.B., O.Y., T.T., T.W., K.H.C. and U.R.A. All authors have read and agreed to the published version of the manuscript.

Funding: This study was supported in part by a University Malaya Research Grant (grant no.: CSRG002 -2020ST) and by the Singapore University of Technology and Design (grant no.: SRG SCI 2019 142).

Institutional Review Board Statement: This research has been approved on ethical grounds by the Medical Research Ethics Committee, University Malaya Medical Centre on 19 June 2020 (2020417-8530).

Informed Consent Statement: Informed consent was obtained from patient partners and participants prior to the interviews and focus groups. 
Data Availability Statement: The data are not publicly available due to restrictions regarding the Ethical Committee Institution.

Acknowledgments: We gratefully acknowledge the Medical Research Ethics Committee, University Malaya Medical Centre, for data transcription.

Conflicts of Interest: The authors declare no conflict of interest.

\section{References}

1. Hu, S.; Gao, Y.; Niu, Z.; Jiang, Y.; Li, L.; Xiao, X.; Wang, M.; Fang, E.F.; Menpes-Smith, W.; Xia, J. Weakly supervised deep learning for COVID-19 infection detection and classification from ct images. IEEE Access 2020, 8, 118869-118883. [CrossRef]

2. Waheed, A.; Goyal, M.; Gupta, D.; Khanna, A.; Al-Turjman, F.; Pinheiro, P.R. Covidgan: Data augmentation using auxiliary classifier gan for improved COVID-19 detection. IEEE Access 2020, 8, 91916-91923. [CrossRef] [PubMed]

3. Cheong, K.H.; Jones, M.C. Introducing the 21st century's New four horsemen of the coronapocalypse. BioEssays 2020, $42,2000063$. [CrossRef] [PubMed]

4. Cheong, K.H.; Wen, T.; Lai, J.W. Relieving Cost of Epidemic by Parrondo's Paradox: A COVID-19 Case Study. Adv. Sci. 2020, 7, 2002324. [CrossRef]

5. Lai, J.W.; Cheong, K.H. Superposition of COVID-19 waves, anticipating a sustained wave, and lessons for the future. BioEssays 2020, 42, 2000178. [CrossRef]

6. Babajanyan, S.; Cheong, K.H. Age-structured SIR model and resource growth dynamics: A COVID-19 study. Nonlinear Dyn. 2021, $1-12$.

7. Alizadehsani, R.; Alizadeh Sani, Z.; Behjati, M.; Roshanzamir, Z.; Hussain, S.; Abedini, N.; Hasanzadeh, F.; Khosravi, A.; Shoeibi, A.; Roshanzamir, M. Risk factors prediction, clinical outcomes, and mortality in COVID-19 patients. J. Med. Virol. 2021, 93, $2307-2320$. [CrossRef]

8. Coronavirus Disease (COVID-19) Pandemic. Available online: https:/ /www.who.int/ (accessed on 1 June 2021).

9. Jaiswal, A.; Gianchandani, N.; Singh, D.; Kumar, V.; Kaur, M. Classification of the COVID-19 infected patients using DenseNet201 based deep transfer learning. J. Biomol. Struct. Dyn. 2020, 1-8. [CrossRef]

10. Wang, W.; Xu, Y.; Gao, R.; Lu, R.; Han, K.; Wu, G.; Tan, W. Detection of SARS-CoV-2 in different types of clinical specimens. JAMA 2020, 323, 1843-1844. [CrossRef]

11. Abbas, A.; Abdelsamea, M.M.; Gaber, M.M. Classification of COVID-19 in chest X-ray images using DeTraC deep convolutional neural network. Appl. Intell. 2021, 51, 854-864. [CrossRef]

12. Quintero, F.O.L.; Contreras-Reyes, J.E. Estimation for finite mixture of simplex models: Applications to biomedical data. Stat. Model. 2018, 18, 129-148. [CrossRef]

13. Zhang, Z.; Shen, Y.; Wang, H.; Zhao, L.; Hu, D. High-resolution computed tomographic imaging disclosing COVID-19 pneumonia: A powerful tool in diagnosis. J. Infect. 2020, 81, 318. [CrossRef] [PubMed]

14. Chen, H.; Ai, L.; Lu, H.; Li, H. Clinical and imaging features of COVID-19. Radiol. Infect. Dis. 2020, 7, 43-50. [CrossRef] [PubMed]

15. Pereira, R.M.; Bertolini, D.; Teixeira, L.O.; Silla, C.N., Jr.; Costa, Y.M. COVID-19 identification in chest X-ray images on flat and hierarchical classification scenarios. Comput. Methods Programs Biomed. 2020, 194, 105532. [CrossRef]

16. Punn, N.S.; Agarwal, S. Automated diagnosis of COVID-19 with limited posteroanterior chest X-ray images using fine-tuned deep neural networks. Appl. Intell. 2021, 51, 2689-2702. [CrossRef]

17. Akilan, T.; Wu, Q.J.; Zhang, H. Effect of fusing features from multiple DCNN architectures in image classification. IET Image Process. 2018, 12, 1102-1110. [CrossRef]

18. Ma, L.; Jiang, W.; Jie, Z.; Jiang, Y.-G.; Liu, W. Matching image and sentence with multi-faceted representations. IEEE Trans. Circuits Syst. Video Technol. 2019, 30, 2250-2261. [CrossRef]

19. Zhang, W.; Wu, Q.J.; Yang, Y.; Akilan, T. Multimodel Feature Reinforcement Framework Using Moore-Penrose Inverse for Big Data Analysis. IEEE Trans. Neural Netw. Learn. Syst. 2020. [CrossRef] [PubMed]

20. Huynh-The, T.; Hua, C.-H.; Kim, D.-S. Encoding pose features to images with data augmentation for 3-D action recognition. IEEE Trans. Ind. Inform. 2019, 16, 3100-3111. [CrossRef]

21. Pahuja, G.; Nagabhushan, T. A comparative study of existing machine learning approaches for parkinson's disease detection. IETE J. Res. 2021, 67, 4-14. [CrossRef]

22. Deivasigamani, S.; Senthilpari, C.; Yong, W.H. Machine learning method based detection and diagnosis for epilepsy in EEG signal. J. Ambient Intell. Humaniz. Comput. 2021, 12, 4215-4221. [CrossRef]

23. Tuncer, T.; Dogan, S.; Pławiak, P.; Acharya, U.R. Automated arrhythmia detection using novel hexadecimal local pattern and multilevel wavelet transform with ECG signals. Knowl. Based Syst. 2019, 186, 104923. [CrossRef]

24. Tuncer, T.; Dogan, S.; Subasi, A. Surface EMG signal classification using ternary pattern and discrete wavelet transform based feature extraction for hand movement recognition. Biomed. Signal Process. Control 2020, 58, 101872. [CrossRef]

25. Jahmunah, V.; Sudarshan, V.K.; Oh, S.L.; Gururajan, R.; Gururajan, R.; Zhou, X.; Tao, X.; Faust, O.; Ciaccio, E.J.; Ng, K.H. Future IoT tools for COVID-19 contact tracing and prediction: A review of the state-of-the-science. Int. J. Imaging Syst. Technol. 2021, 31, 455-471. [CrossRef] [PubMed] 
26. Sharifrazi, D.; Alizadehsani, R.; Roshanzamir, M.; Joloudari, J.H.; Shoeibi, A.; Jafari, M.; Hussain, S.; Sani, Z.A.; Hasanzadeh, F.; Khozeimeh, F. Fusion of convolution neural network, support vector machine and Sobel filter for accurate detection of COVID-19 patients using X-ray images. Biomed. Signal Process. Control 2021, 68, 102622. [CrossRef]

27. Abdar, M.; Salari, S.; Qahremani, S.; Lam, H.-K.; Karray, F.; Hussain, S.; Khosravi, A.; Acharya, U.R.; Nahavandi, S. UncertaintyFuseNet: Robust Uncertainty-aware Hierarchical Feature Fusion with Ensemble Monte Carlo Dropout for COVID-19 Detection. arXiv 2021, arXiv:2105.08590.

28. Wang, S.; Zha, Y.; Li, W.; Wu, Q.; Li, X.; Niu, M.; Wang, M.; Qiu, X.; Li, H.; Yu, H. A fully automatic deep learning system for COVID-19 diagnostic and prognostic analysis. Eur. Respir. J. 2020, 56, 2000775. [CrossRef] [PubMed]

29. Jamshidi, M.; Lalbakhsh, A.; Talla, J.; Peroutka, Z.; Hadjilooei, F.; Lalbakhsh, P.; Jamshidi, M.; La Spada, L.; Mirmozafari, M.; Dehghani, M. Artificial intelligence and COVID-19: Deep learning approaches for diagnosis and treatment. IEEE Access 2020, 8 , 109581-109595. [CrossRef] [PubMed]

30. Krizhevsky, A.; Sutskever, I.; Hinton, G.E. Imagenet classification with deep convolutional neural networks. Adv. Neural Inf. Process. Syst. 2012, 25, 1097-1105. [CrossRef]

31. Simonyan, K.; Zisserman, A. Very deep convolutional networks for large-scale image recognition. arXiv 2014, arXiv:1409.1556.

32. Tuncer, T.; Dogan, S.; Özyurt, F.; Belhaouari, S.B.; Bensmail, H. Novel Multi Center and Threshold Ternary Pattern Based Method for Disease Detection Method Using Voice. IEEE Access 2020, 8, 84532-84540. [CrossRef]

33. Vapnik, V. The support vector method of function estimation. In Nonlinear Modeling; Springer: Berlin, Germany, $1998 ;$ pp. 55-85.

34. Vapnik, V. The Nature of Statistical Learning Theory; Springer Science \& Business Media: Berlin, Germany, 2013.

35. Narin, A.; Kaya, C.; Pamuk, Z. Automatic detection of coronavirus disease (COVID-19) using X-ray images and deep convolutional neural networks. Pattern Anal. Appl. 2021, 1-14. [CrossRef]

36. Muhammad, G.; Hossain, M.S. COVID-19 and non-COVID-19 classification using multi-layers fusion from lung ultrasound images. Inf. Fusion 2021, 72, 80-88. [CrossRef] [PubMed]

37. Loey, M.; Smarandache, F.; Khalifa, N.E. Within the lack of chest COVID-19 X-ray dataset: A novel detection model based on GAN and deep transfer learning. Symmetry 2020, 12, 651. [CrossRef]

38. Saad, W.; Shalaby, W.A.; Shokair, M.; Abd El-Samie, F.; Dessouky, M.; Abdellatef, E. COVID-19 classification using deep feature concatenation technique. J. Ambient Intell. Humaniz. Comput. 2021, 1-19. [CrossRef]

39. Tuncer, T.; Dogan, S.; Ozyurt, F. An automated Residual Exemplar Local Binary Pattern and iterative ReliefF based COVID-19 detection method using chest X-ray image. Chemom. Intell. Lab. Syst. 2020, 203, 104054. [CrossRef]

40. Sharma, V.; Dyreson, C. COVID-19 detection using residual attention network an artificial intelligence approach. arXiv 2020, arXiv:2006.16106.

41. Jia, G.; Lam, H.-K.; Xu, Y. Classification of COVID-19 chest X-Ray and CT images using a type of dynamic CNN modification method. Comput. Biol. Med. 2021, 134, 104425. [CrossRef] [PubMed]

42. Bassi, P.R.; Attux, R. A deep convolutional neural network for COVID-19 detection using chest X-rays. Res. Biomed. Eng. 2021, $1-10$.

43. Goldberger, J.; Hinton, G.E.; Roweis, S.; Salakhutdinov, R.R. Neighbourhood components analysis. Adv. Neural Inf. Process. Syst. 2004, 17, 513-520.

44. Dosovitskiy, A.; Beyer, L.; Kolesnikov, A.; Weissenborn, D.; Zhai, X.; Unterthiner, T.; Dehghani, M.; Minderer, M.; Heigold, G.; Gelly, S. An image is worth 16x16 words: Transformers for image recognition at scale. arXiv 2020, arXiv:2010.11929.

45. Shoeibi, A.; Khodatars, M.; Alizadehsani, R.; Ghassemi, N.; Jafari, M.; Moridian, P.; Khadem, A.; Sadeghi, D.; Hussain, S.; Zare, A. Automated detection and forecasting of COVID-19 using deep learning techniques: A review. arXiv 2020, arXiv:2007.10785.

46. Kermany, D.; Zhang, K.; Goldbaum, M. Large Dataset of Labeled Optical Coherence Tomography (OCT) and Chest X-ray Images. Available online: https:/ / data.mendeley.com/datasets/rscbjbr9sj/3 (accessed on 1 April 2021).

47. Kermany, D.S.; Goldbaum, M.; Cai, W.; Valentim, C.C.; Liang, H.; Baxter, S.L.; McKeown, A.; Yang, G.; Wu, X.; Yan, F. Identifying medical diagnoses and treatable diseases by image-based deep learning. Cell 2018, 172, 1122-1131.e1129. [CrossRef] [PubMed]

48. Ozturk, T.; Talo, M.; Yildirim, E.A.; Baloglu, U.B.; Yildirim, O.; Acharya, U.R. Automated detection of COVID-19 cases using deep neural networks with X-ray images. Comput. Biol. Med. 2020, 121, 103792. [CrossRef]

49. Github. COVID-19. Available online: https://github.com/muhammedtalo/COVID-19 (accessed on 14 March 2021).

50. Github. COVID Chestxray Dataset. Available online: https://github.com/ieee8023/covid-chestxray-dataset/tree/master/images (accessed on 14 March 2021).

51. Rahman, T. COVID-19 Radiography Database. Available online: https://www.kaggle.com/tawsifurrahman/covid19-radiographydatabase (accessed on 21 April 2021).

52. Chowdhury, M.E.; Rahman, T.; Khandakar, A.; Mazhar, R.; Kadir, M.A.; Mahbub, Z.B.; Islam, K.R.; Khan, M.S.; Iqbal, A.; Al Emadi, N. Can AI help in screening viral and COVID-19 pneumonia? IEEE Access 2020, 8, 132665-132676. [CrossRef]

53. Rahman, T.; Khandakar, A.; Qiblawey, Y.; Tahir, A.; Kiranyaz, S.; Kashem, S.B.A.; Islam, M.T.; Al Maadeed, S.; Zughaier, S.M.; Khan, M.S. Exploring the effect of image enhancement techniques on COVID-19 detection using chest X-ray images. Comput. Biol. Med. 2021, 132, 104319. [CrossRef]

54. Murugan, R.; Goel, T. E-DiCoNet: Extreme learning machine based classifier for diagnosis of COVID-19 using deep convolutional network. J. Ambient Intell. Humaniz. Comput. 2021, 1-12. [CrossRef] 
55. Gilanie, G.; Bajwa, U.I.; Waraich, M.M.; Asghar, M.; Kousar, R.; Kashif, A.; Aslam, R.S.; Qasim, M.M.; Rafique, H. Coronavirus (COVID-19) detection from chest radiology images using convolutional neural networks. Biomed. Signal Process. Control 2021, 66, 102490. [CrossRef]

56. Pandit, M.K.; Banday, S.A.; Naaz, R.; Chishti, M.A. Automatic detection of COVID-19 from chest radiographs using deep learning. Radiography 2021, 27, 483-489. [CrossRef]

57. Nigam, B.; Nigam, A.; Jain, R.; Dodia, S.; Arora, N.; Annappa, B. COVID-19: Automatic detection from X-ray images by utilizing deep learning methods. Expert Syst. Appl. 2021, 176, 114883. [CrossRef]

58. Hussain, E.; Hasan, M.; Rahman, M.A.; Lee, I.; Tamanna, T.; Parvez, M.Z. CoroDet: A deep learning based classification for COVID-19 detection using chest X-ray images. Chaos Solitons Fractals 2021, 142, 110495. [CrossRef] [PubMed]

59. Shi, W.; Tong, L.; Zhu, Y.; Wang, M.D. COVID-19 Automatic Diagnosis with Radiographic Imaging: Explainable AttentionTransfer Deep Neural Networks. IEEE J. Biomed. Health Inform. 2021, 25, 2376-2387. [CrossRef] [PubMed]

60. Mukherjee, H.; Ghosh, S.; Dhar, A.; Obaidullah, S.M.; Santosh, K.; Roy, K. Deep neural network to detect COVID-19: One architecture for both CT Scans and Chest X-rays. Appl. Intell. 2021, 51, 2777-2789. [CrossRef]

61. Sitaula, C.; Hossain, M.B. Attention-based VGG-16 model for COVID-19 chest X-ray image classification. Appl. Intell. 2021, 51, 2850-2863. [CrossRef] 